\title{
Multimorbilidad y síndromes geriátricos Su efecto sobre la mortalidad en adultos mayores con sepsis
}

\author{
Multimorbidity and geriatric syndromes \\ Their effect on mortality in older adults with sepsis
}

\author{
Jenny Patricia Muñoz-Lombo, Adrián Tabares-Burbano, José Mauricio Ocampo-Chaparro, \\ Reynaldo Carvajal-Ortiz, María Eugenia Casanova-Valderrama. Cali (Colombia) \\ Carlos Alfonso Reyes-Ortiz • Tallahassee (USA)
}

DOI: https://doi.org/10.36104/amc.2022.2125

\section{Resumen}

Introducción: la sepsis se diagnostica en más de $60 \%$ de los adultos mayores (AM) en el mundo. Estos AM con frecuencia presentan multimorbilidad y alguno de los síndromes geriátricos, llevando a discapacidad física, cognitiva y psicosocial, lo cual produce altos costos para los sistemas de salud, resultando en un problema grave de salud pública.

Objetivo: identificar el impacto de la multimorbilidad y los síndromes geriátricos en la mortalidad de AM hospitalizados por sepsis en una unidad geriátrica de agudos a 30 días de su ingreso.

Material y métodos: estudio observacional, analítico de casos y controles anidado en una cohorte.

Resultados: se analizaron 238 pacientes con edad media de $83.15 \pm 7.12$ años, $52.1 \%$ fueron mujeres y el $99 \%$ tenían al menos una comorbilidad, la mortalidad a 30 días fue 34\%. La infección urinaria fue la causa principal de hospitalización (42.9\%), obteniendo un aislamiento microbiológico en $43.3 \%$ de los casos, siendo la Escherichia coli el agente causal más frecuente (46.6\%). La regresión logística múltiple mostró que la enfermedad renal crónica (OR 2.1 IC 95\% 1.1-4.8; $\mathrm{p}=0.037$ ), el delirium (OR 3.1 IC 95\% 1.6-5.8; p=0.001) y la discapacidad (índice de Barthel <60; OR 3.4 IC $95 \%$ 1.5-7.5; $\mathrm{p}=0.002$ ) se asociaron de manera significativa con la mortalidad a 30 días desde el ingreso a la unidad geriátrica de agudos en paciente con sepsis.

Conclusión: en los AM hospitalizados por sepsis, la multimorbilidad, la enfermedad renal crónica y los síndromes geriátricos representados por delirium y discapacidad fueron los predictores de mortalidad a 30 días. (Acta Med Colomb 2022; 47. DOI: https://doi.org/10.36104/ amc.2022.2125).

Palabras clave: hospitalización, sepsis, síndrome geriátrico, multimorbilidad, valoración geriátrica integral, mortalidad.

\section{Abstract}

Introduction: sepsis is diagnosed in more than $60 \%$ of older adults (OAs) worldwide. These OAs often have multimorbidity and one of the geriatric syndromes, leading to physical, cognitive and psychosocial disability with consequently high healthcare costs, resulting in a serious public health problem.

Objective: to determine the impact of multimorbidity and geriatric syndromes on the 30-day mortality rate of OAs hospitalized for sepsis in an acute geriatric unit

Materials and methods: an observational, analytical, nested case-control study.

Results: 238 patients with a mean age of $83.15 \pm 7.12$ were analyzed; $52.1 \%$ were women and $99 \%$ had at least one comorbidity; the 30-day mortality was $34 \%$. Urinary tract infection was the main cause of hospitalization (42.9\%), with microbiological isolation achieved in $43.3 \%$ of cases and Escherichia coli being the most common causal agent (46.6\%). Multiple logistic regression showed that chronic kidney disease (OR 2.1 95\% CI 1.1-4.8; $\mathrm{p}=0.037$ ), delirium (OR $3.195 \%$ CI 1.6-5.8; $\mathrm{p}=0.001$ ) and disability (Barthel index <60; OR 3.4 95\% CI 1.5-7.5; $\mathrm{p}=0.002$ ) were significantly related to 30 -day mortality in patients with sepsis admitted to an acute geriatric unit.
Dres. Jenny Patricia Muñoz-Lombo, Adrián Tabares-Burbano, Reynaldo Carvajal-Ortiz, María Eugenia Casanova-Valderrama: Universidad Libre, Facultad Ciencias de la Salud, Departamento de Medicina Interna, Grupo Interinstitucional de Medicina Interna (GIMI 1); Dr. José Mauricio Ocampo-Chaparro: Universidad Libre, Facultad Ciencias de la Salud, Departamento de Medicina Interna, Grupo Interinstitucional de Medicina Interna (GIMI 1). Universidad del Valle, Facultad de Salud, Departamento Medicina Familiar. Cali (Colombia); Dr. Carlos Alfonso Reyes-Ortiz: PhD. Institute of Public Health, Behavioral Sciences \& Health Education, College of Pharmacy and Pharmaceutical Sciences, Florida $A \& M$ University. Tallahassee, Florida (USA).

Correspondencia: Dr. Carlos A. Reyes-Ortiz. Tallahassee, Florida (USA).

E-Mail: carlosareyesort@gmail.com, carlos. reyesortiz@famu.edu

Recibido: 26/II/2021 Aceptado: 09/IX/2021 
Conclusion: in OAs hospitalized for sepsis, multimorbidity, chronic kidney disease and geriatric syndromes (represented by delirium and disability) were the predictors of 30-day mortality. (Acta Med Colomb 2022; 47. DOI: https://doi.org/10.36104/amc.2022.2125).

Key words: hospitalization, sepsis, geriatric syndrome, multimorbidity, comprehensive geriatric assessment, mortality.

\section{Introducción}

La sepsis es definida como una disfunción orgánica potencialmente mortal causada por la respuesta del huésped a la infección ya sea en un tejido sano o con una lesión tisular, generando alteraciones fisiopatológicas y bioquímicas que representan un amplio espectro de manifestaciones clínicas y paraclínicas que van desde la bacteriemia hasta el choque séptico (1); un diagnóstico temprano, tratamiento adecuado y oportuno son la clave para limitar el daño y controlar la enfermedad (2).

Es considerada como una de las condiciones de mayor carga de morbimortalidad del siglo XXI, la sepsis afecta con alta frecuencia a adultos mayores (AM) en quienes a pesar de los avances en el diagnóstico y tratamiento, su mortalidad general sigue siendo alta, causando más del $60 \%$ de las muertes hospitalarias en mayores de 65 años y hasta $80 \%$ en los mayores o iguales a 80 años (3-5).

Existen varios factores que predisponen al desarrollo de la sepsis en el AM, producto de los cambios propios del envejecimiento, como el estrés oxidativo, la supresión mitocondrial, el acortamiento de los telómeros y el daño del ADN que generan a nivel celular alteraciones en la regulación de las citoquinas y en la respuesta del sistema neuroendocrino con modificaciones en el metabolismo de las proteínas que asociados con baja ingesta conllevan a la desnutrición y cambios de la microbiota intestinal, potenciando un estado proinflamatorio e inmunosenescencia que pueden generar o empeorar condiciones neurológicas que desencadenan deterioro del estado funcional con atrofia por desuso, sarcopenia por pérdida muscular acelerada, lentitud y fatiga (6).

Por otro lado los AM padecen con gran frecuencia de multimorbilidad, definida como la concurrencia de múltiples condiciones crónicas que requieren rehabilitación o un largo periodo de atención sanitaria (7), que en muchas ocasiones se encuentra en estadio avanzado, siendo un factor de riesgo importante para el desarrollo de enfermedades infecciosas particularmente neumonía, infección urinaria y de piel y tejidos blandos (8). Estas infecciones pueden debutar con sintomatología atípica como el delirium, la declinación funcional, la pérdida de peso, la fatiga, el mareo y las caídas, síntomas que hacen parte de los síndromes geriátricos y que generan desafíos especiales en el diagnóstico, tratamiento de esta población, el cual debe ser ofrecido por un equipo interdisciplinario $(7,9)$ el modelo de trabajo en equipo interdisciplinario, la asistencia al paciente en diferentes niveles asistenciales (unidad de agudos, de media estancia, hospital de día, atención domiciliaria).

Según reportes epidemiológicos de los Estados Unidos, a pesar de los avances médicos, de mayores accesos a los servicios de salud y del mejoramiento de la calidad de vida, la sepsis continua generando mortalidad en los pacientes hospitalizados hasta en 30\% (3), que puede llegar hasta el $50 \%$ si hay presencia de choque séptico, porcentajes comparables con la mortalidad ocasionada por enfermedades cardiovasculares (10). De los sobrevivientes, la gran mayoría desarrollan complicaciones médicas, mentales y funcionales que llevan a discapacidad física, cognitiva y psicosocial $(11,12)$, con altos costos económicos para los sistemas de salud (13) traduciéndose en un problema de interés en salud pública (4). En el año 2017, Colombia reportó 224187 muertes de las cuales 62\% fue mayores de 65 años; siendo la sepsis la quinta causa de mortalidad a nivel nacional (14). Por lo anterior, el objetivo de la investigación fue identificar el efecto que tiene la multimorbilidad y los síndromes geriátricos sobre la mortalidad en AM hospitalizados por sepsis en una unidad geriátrica de agudos.

\section{Diseño}

\section{Material y métodos}

Se realizó un estudio observacional, analítico de casos y controles anidados en una cohorte de pacientes atendidos en el servicio de hospitalización de una unidad geriátrica de agudos a quienes se les realizó un diagnóstico de sepsis.

El protocolo del estudio fue aprobado por el Comité Técnico-Científico y Bioético de la Universidad Libre y de la Clínica Universitaria Rafael Uribe Uribe como una investigación sin riesgo, los pacientes o algunos cuidadores se entrevistaron para obtener la información durante la hospitalización. Se manifiesta no tener ningún tipo de conflicto de intereses.

\section{Población de estudio}

De la cohorte de 2054 pacientes AM de 60 años o más que fueron atendidos en la Corporación Universitaria Rafael Uribe Uribe (CURUU) en Cali (Colombia) entre enero del 2012 hasta septiembre del 2015, todos cumplieron con al menos uno de los siguientes criterios de inclusión para ser manejados en la unidad geriátrica de agudos (UGA): consumo de ocho o más fármacos, evento cerebrovascular en etapas subagudas, enfermedades incapacitantes o condición funcional previa de dependencia severa o 
total, readmisiones hospitalarias frecuentes (dos o más al mes), condiciones mentales previas (delirium al ingreso o demencia de base), presencia de múltiples síndromes geriátricos (fragilidad, úlceras por presión, caídas), índice de masa corporal menor de $20 \mathrm{~kg} / \mathrm{m}^{2}$, red de apoyo social insuficiente, procedencia de instituciones geriátricas o pacientes mayores de 80 años con enfermedad médica aguda. De la cohorte de 2054 pacientes se analizaron los registros con diagnóstico de sepsis definiendo como casos todos los registros en quien se presentó mortalidad a los 30 días desde el momento del ingreso y como controles a los sobrevivientes. El pareamiento se realizó un caso por cada dos controles.

El consentimiento informado fue obtenido de cada paciente o por un familiar o cuidador en el caso que el AM tuviera la incapacidad de diligenciarlo. Posteriormente se procedió aplicar el cuestionario que fue completado al momento del ingreso hospitalario por un médico del equipo interdisciplinario con entrenamiento para recolectar la información estandarizada de escalas de valoración geriátrica integral (VGI).

La información obtenida fue extraída de la base de datos del equipo interdisciplinario de geriatría quienes evaluaron e intervinieron al paciente, familia y cuidadores fundamentados en el modelo biopsicosocial durante su estancia hospitalaria. Así cada profesional involucrado realizó la aplicación de escalas de clinimetría geriátrica según su área disciplinar, médico geriatra: información relacionada con la esfera biológica (causa de hospitalización, comorbilidad, muerte, medicamentos), fisioterapeuta: información relacionada con la esfera funcional (actividades de la vida diaria), psicóloga: información relacionada con la esfera mental (depresión, delirium y deterioro cognoscitivo) y trabajadora social: información relacionada con la esfera social y familiar (variables sociodemográficas, presencia de cuidador, relación con el cuidador y condición sociofamiliar).

\section{Desenlace}

Mortalidad a 30 días desde el ingreso a la UGA. La información de mortalidad fue suministrada por la Secretaría de Salud Departamental del Valle del Cauca mediante los certificados de defunción hasta octubre del año 2015.

\section{Variable independiente principal}

La sepsis se diagnosticó basado en la presencia de signos y síntomas clínicos de respuesta inflamatoria sistémica como respuesta del huésped a la infección asociado con marcadores biológicos de laboratorio dados como leucocitosis, neutrofilia, proteína $\mathrm{C}$ reactiva y aislamiento microbiológico cuando fue posible el caso, de igual manera el apoyo de ayudas imagenológicas con hallazgos compatibles de un proceso infeccioso $(1,15)$. Es importante tener en cuenta que el estudio fue llevado a cabo en una unidad hospitalaria y no de cuidados intermedios o intensivos donde variables como el SOFA o el APACHE no son evaluadas de rutina para determinar la gravedad de los pacientes.

\section{Covariables}

Se incluyeron en los análisis la edad, el sexo y las variables conocidas que pudieran afectar el desenlace, los valores de laboratorio se usaron como variables numéricas. Se definió al modelo de multimorbilidad como la presencia de dos o más condiciones crónicas, que no se pueden curar pero que se pueden controlar mediante medicamentos u otros tratamientos, o sea enfermedades crónicas (16). Para la valoración del estado funcional se utilizó el índice de Barthel (IB) que evalúa 10 actividades básicas de la vida diaria asignándole un valor predeterminado para la autonomía/independencia al ingreso; la puntuación varía de 0 a 100, siendo 0 la máxima dependencia y 100 la independencia total. El IB se usó como variable numérica y categórica el cual se ha recomendado como un instrumento de elección para la medida de la discapacidad física. Un valor en el IB<60 se consideró como discapacidad $(17,18)$, que se definió como la dificultad o limitación para realizar las actividades básicas de la vida diaria (19).

Para la evaluación de delirium se empleó el método CAM (Confussion Assessment Method), que evalúa cuatro características: inicio agudo y curso fluctuante, falta de atención, pensamiento desorganizado y nivel de conciencia alterado, considerándose positivo para delirium la presencia de las características 1 y 2, y 3 o 4 (20). La función renal fue evaluada mediante la estimación de la tasa de filtración glomerular (TFG) empleando la ecuación derivada del estudio de Levey et al. (MDRD-4 [Modification of Diet in Renal Disease Study equation] o MDRD-IDMS) (21) y se definió enfermedad renal crónica (ERC) como una TFG inferior a $60 \mathrm{ml} / \mathrm{min} / 1,73 \mathrm{~m}^{2} \mathrm{o}$ lesión renal presentes durante tres meses o más, basados en la definición de KDIGO del 2013. Para la definición de ERC no se tuvieron en cuenta marcadores de lesión renal (22).

\section{Plan de análisis}

Para resumir las características de la población del estudio se usó estadística descriptiva. Las variables cuantitativas se presentaron a través de sus medidas de tendencia central y dispersión. Para contrastar la distribución de los datos se usó la prueba de Kolmogórov-Smirnov. Las variables cualitativas se resumieron en porcentaje y se presentaron en tablas de frecuencia. Para evaluar la significancia estadística de las asociaciones bivariadas con la variable desenlace (mortalidad a 30 días), se realizó la prueba U de Mann Whitney para las variables numéricas y para las variables dicotómicas se realizó $\mathrm{chi}^{2}$ o Fisher según correspondiera, asumiendo como significante para rechazar las hipótesis nulas un valor de $\mathrm{p} \leq 0.05$.

Para explorar la asociación multivariada entre las variables de exposición y la variable desenlace, se usó como 
medida de asociación la Odds Ratio (OR) con sus respectivos intervalos de confianza de 95\% (IC95\%).

Se definieron los modelos de morbimortalidad y de síndromes geriátricos como construcciones teóricas que engloban un conjunto de aspectos relacionados entre sí. Para la creación de estos modelos se incluyeron las variables que en análisis bivariado presentaron una significancia estadística $<0.5$. Toda la información fue registrada en Excel y analizada con el software IBMSPSS versión 20.0.

\section{Resultados}

De la evaluación de los 2054 registros de pacientes atendidos en la UGA de la Corporación Universitaria Rafael Uribe Uribe (CRUU), se seleccionaron 238 que cumplieron con los criterios de inclusión para ser analizados en este estudio, las razones para la exclusión se muestran en la Figura 1.

La edad promedio fue de $83.2 \pm 7$, un año, siendo el $52.1 \%$ mujeres. El 99\% de los pacientes cursaban con alguna enfermedad crónica, siendo la hipertensión arterial (73.9\%) y la diabetes mellitus (31.1\%) las más frecuentes; de igual manera se observa que la enfermedad cerebrovascular (ECV) y la ERC presentaron diferencias estadísticamente significativas entre ambos grupos a favor de los casos (pacientes fallecidos), siendo limítrofe para enfermedad coronaria (Tabla 1). En el análisis bivariado para el modelo de síndromes geriátricos, se documentaron diferencias estadísticamente significativas para mortalidad a 30 días desde el ingreso a la UGA del $48 \%$ para casos con delirium y $87 \%$ para casos con discapacidad (Tabla 1).

En relación con el sitio de infección, se observó que la infección del tracto urinario fue la causa de hospitalización más frecuente, seguido por neumonía y la infección de tejidos blandos con 42.9, 38.7 y $16.0 \%$ respectivamente. El aislamiento microbiológico se obtuvo en $43.2 \%$ de los casos, de los cuales el $100 \%$ pertenece a la familia de los Enterobacteriales, siendo Escherichia coli el agente causal más frecuente en un $46.6 \%$ con un patrón de resistencia natural en $11.8 \%$ (Tabla 2).

En el modelo de multimorbilidad, el análisis bivariado demostró que la ERC (OR 2.5, IC 95\% 1.2-5.5; p=0.017) y la ECV (OR 2.1, IC 95\% 1.1-4.0; p=0.023) fueron variables estadísticamente significativas (Tabla 3 ). En cuanto al modelo de síndromes geriátricos fueron estadísticamente significativos el delirium (OR 4.5, IC 95\% 2.5-8.2; $\mathrm{p}<0.001$ ) y la discapacidad (OR 5.6, IC 95\% 2.7-11.9; $\mathrm{p}<0.001$ ) (Tabla 4).

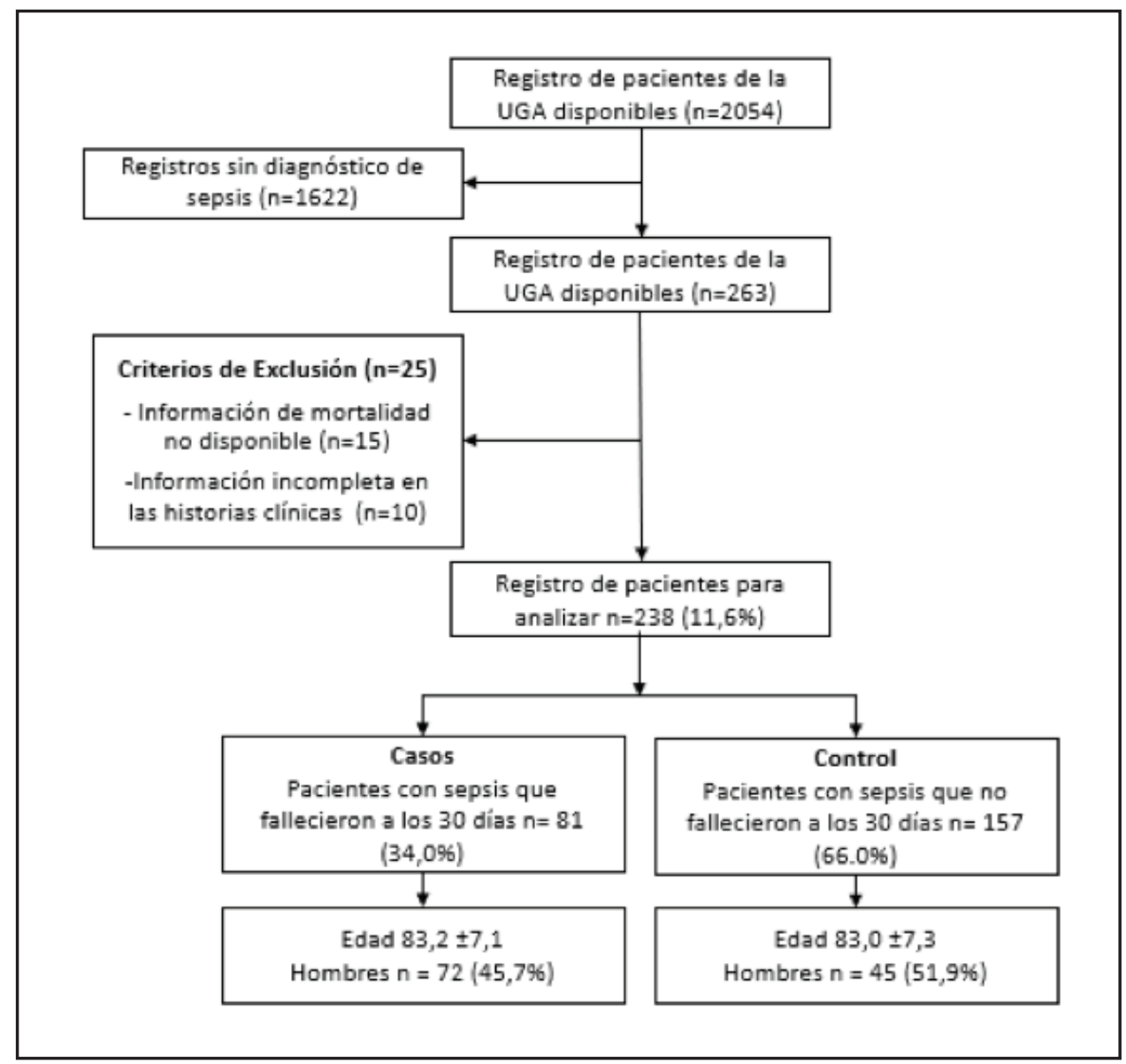

Figura 1. Diagrama de flujo del estudio. 
TRABAJOS ORIGINALES • Adultos mayores con sepsis

En el análisis de regresión múltiple, donde se ingresaron todas las variables que fueron significativas en los modelos de multimorbilidad y síndromes geriátricos, se documentó que la ERC (OR 2.1, IC 95\% 1.1-4.8, p=0.037), el delirium
(OR 3.1 IC 95\% 1.6-5.8; $\mathrm{p}=0.001$ ) y la discapacidad (OR 3.4 IC 95\% 1.5-7.5; $\mathrm{p}=0.002$ ) se asociaron de manera estadísticamente significativa con la mortalidad en AM hospitalizados por sepsis en una UGA a 30 días de su ingreso (Tabla 5).

Tabla 1. Características sociodemográficas, mentales y funcionales de la población estudiada.

\begin{tabular}{|c|c|c|c|c|}
\hline \multirow{2}{*}{ Características } & \multirow{2}{*}{$\begin{array}{c}\text { General } \\
\mathbf{n}(\%)(\mathbf{n}=238)\end{array}$} & \multicolumn{2}{|c|}{ Estado después del diagnóstico de sepsis } & \multirow{2}{*}{ Valor $\mathbf{p}$} \\
\hline & & Fallecido $n(\%)(n=81)$ & Vivo $n(\%) n=157$ & \\
\hline Edad* & $83.15 \pm 7.12$ & $83.02 \pm 7.31$ & $83.22 \pm 7.05$ & $0.84^{t}$ \\
\hline Masculino & $114(47.90)$ & $42(51.85)$ & $72(45.86)$ & 0.38 \\
\hline HTA $(n=234)$ & $173(73.93)$ & $61(75.31)$ & $112(73.20)$ & 0.73 \\
\hline Hipotiroidismo & $35(14.71)$ & $10(12.35)$ & $25(15.92)$ & 0.46 \\
\hline Diabetes mellitus & $74(31.09)$ & $25(30.86)$ & $49(31.21)$ & 0.96 \\
\hline Insuficiencia cardiaca congestiva & $27(11.34)$ & $10(12.35)$ & $17(10.83)$ & 0.73 \\
\hline Enfermedad coronaria & $16(6.72)$ & $9(11.11)$ & $7(4.46)$ & 0.05 \\
\hline Enfermedad cerebrovascular & $48(20.17)$ & $23(28.40)$ & $25(15.92)$ & 0.02 \\
\hline Fibrilación auricular & $16(6.72)$ & $7(8.64)$ & $9(5 . .3)$ & 0.40 \\
\hline Enfermedad renal crónica & $30(12.61)$ & $16(19.75)$ & $14(8.92)$ & 0.02 \\
\hline Enfermedad arterial oclusiva & $11(4.62)$ & $5(6.17)$ & $6(3.82)$ & $0.52^{\mathrm{f}}$ \\
\hline Multimorbilidad & $236(99.16)$ & $80(98.77)$ & $156(99.36)$ & 1.00 \\
\hline Anemia & $9(3.78)$ & $4(4.94)$ & $5(3.18)$ & $0.49^{\mathrm{f}}$ \\
\hline Demencia & $43(18.07)$ & $17(20.99)$ & $26(16.56)$ & 0.40 \\
\hline Neoplasias & $22(9.24)$ & $9(11.11)$ & $13(8.28)$ & 0.48 \\
\hline Enfermedad pulmonar obstructiva crónica & 49 (20.59) & $16(19.75)$ & $33(21.02)$ & 0.82 \\
\hline Polifarmacia & $134(56.30)$ & $50(61.73)$ & $84(53.50)$ & 0.22 \\
\hline Delirium & $66(27.73)$ & $39(48.15)$ & $27(17.20)$ & 0.00 \\
\hline Discapacidad & $158(66.39)$ & $71(87.65)$ & $87(55.41)$ & 0.00 \\
\hline Estancia hospitalaria (días)** & $10(6-15)$ & $11(5-21)$ & $10(7-14)$ & $0.31^{\mathrm{m}}$ \\
\hline
\end{tabular}

Tabla 2. Características de microbiológica encontrada de la población estudiada.

\begin{tabular}{|c|c|c|c|c|}
\hline \multirow{2}{*}{ Características } & \multirow{2}{*}{$\begin{array}{c}\text { General } \\
\mathbf{n}(\%)(\mathbf{n}=\mathbf{2 3 8})\end{array}$} & \multicolumn{2}{|c|}{ Estado después del diagnóstico de sepsis } & \multirow{2}{*}{ Valor $\mathbf{p}$} \\
\hline & & Fallecido n (\%) (n=81) & Vivo $n(\%) n=157$ & \\
\hline Aislamiento microbiológico & $103(43.28)$ & $29(35.80)$ & $74(47.13)$ & 0.10 \\
\hline Escherichia coli patrón natural & $28(11.76)$ & $1(1.23)$ & $27(17.20)$ & 0.00 \\
\hline Escherichia coli BLEE & $20(8.40)$ & $6(7.41)$ & $14(8.92)$ & 0.69 \\
\hline Klebsiella pneumoniae Patrón natural & $7(2.94)$ & $0(0.00)$ & $7(4.46)$ & $0.10^{\mathrm{f}}$ \\
\hline Klebsiella pneumoniae BLEE & $11(4.62)$ & $6(7.41)$ & $5(3.18)$ & $0.19^{f}$ \\
\hline Klebsiella pneumoniae KPC & $3(1.26)$ & $3(3.70)$ & $0(0.00)$ & $0.04^{\mathrm{f}}$ \\
\hline
\end{tabular}


Tabla 3. Factores de riesgo para mortalidad a 30 días por sepsis. Resultados de regresión logística del modelo conformado por variables de multimorbilidad.

\begin{tabular}{|c|c|c|c|c|c|}
\hline Multimorbilidad & $\begin{array}{c}\text { Casos }(\mathrm{n}=81) \\
(\%)\end{array}$ & $\begin{array}{c}\text { Controles } \\
(n=157)(\%)\end{array}$ & OR & IC $(95 \%)$ & Valor $\mathbf{p}$ \\
\hline Enfermedad cerebrovascular & 28.4 & 15.9 & 2.1 & $1.1-4.0$ & 0.023 \\
\hline Enfermedad renal crónica & 19.8 & 8.9 & 2.5 & $1.2-5.5$ & 0.017 \\
\hline Enfermedad coronaria & 11.1 & 4.5 & 2.7 & $0.9-7.5$ & 0.052 \\
\hline Diabetes mellitus & 30.9 & 31.2 & 1.0 & $0.6-1.8$ & 0.956 \\
\hline Hipertensión arterial & 75.3 & 73.2 & 1.1 & $0.6-2.1$ & 0.727 \\
\hline Enfermedad arterial oclusiva & 6.2 & 3.8 & 1.7 & $0.5-5.6$ & 0.671 \\
\hline
\end{tabular}

Tabla 4. Factores de riesgo para mortalidad a 30 días por sepsis. Resultado de la regresión logística de acuerdo con el modelo conformado por las variables de síndrome geriátrico.

\begin{tabular}{|c|c|c|c|c|c|}
\hline Síndrome geriátrico & Casos $(n=81)(\%)$ & $\begin{array}{c}\text { Controles } \\
(n=157)(\%)\end{array}$ & OR & IC $(95 \%)$ & Valor $\mathbf{p}$ \\
\hline Delirium & 48.1 & 17.2 & 4.5 & $2.5-8.2$ & $<.0001$ \\
\hline Demencia & 21.0 & 16.6 & 1.3 & $0.7-2.6$ & 0.400 \\
\hline Discapacidad & 87.7 & 55.4 & 5.7 & $2.7-11.9$ & $<.0001$ \\
\hline Polifarmacia & 75.3 & 73.2 & 1.1 & $0.6-2.1$ & 0.727 \\
\hline
\end{tabular}

Tabla 5. Factores de riesgo para mortalidad a 30 días por sepsis. Resultados de la regresión logística de acuerdo con el modelo final conformado por variables significantes.

\begin{tabular}{|l|c|c|c|}
\hline \multicolumn{1}{|c|}{ Variables } & OR & IC (95\%) & Valor p \\
\hline Enfermedad cerebrovascular & 1.5 & $0.7-3.0$ & 0.274 \\
\hline Enfermedad renal crónica & 2.1 & $1.1-4.8$ & 0.037 \\
\hline Delirium & 3.1 & $1.6-5.8$ & 0.001 \\
\hline Discapacidad & 3.4 & $1.5-7.5$ & 0.002 \\
\hline
\end{tabular}

\section{Discusión}

En el presente estudio evaluamos la presencia de multimorbilidad y síndromes geriátricos como factores de riesgo para mortalidad en una población colombiana de AM hospitalizados por sepsis en una UGA. Tal como se evidencia en los resultados la presencia de ERC, delirium y discapacidad fueron los principales factores asociados con mortalidad a 30 días en AM hospitalizados por sepsis.

Estudios han demostrado que la edad es un factor de riesgo independiente para mortalidad tanto para todas las causas como para sepsis $(5,12)$, sugiriendo además que la multimorbilidad desempeñan un rol importante en este aspecto $(4,23)$, como se observó en un estudio retrospectivo que evaluó los resultados de adultos ingresados con sepsis grave a 171 UCIs en Australia y Nueva Zelanda, reportando una mortalidad en pacientes sin comorbilidades de 14 vs $26.4 \%$ en pacientes con multimorbilidad, lo cual fue estadísticamente significativo $(\mathrm{p}<0.001)$. En esta misma cohorte, los adultos de 44 años o menos tuvieron una mortalidad de 7.3 vs $30.4 \%$ en adultos de 85 años o más $(p<0.001)$ (4). Lo anterior está en concordancia con lo evidenciado en nuestro estudio donde la mortalidad a 30 días a partir del ingreso fue de $34 \%$ y la multimorbilidad estaba presente en más de 99\% de los pacientes.

Por otro lado se ha evidenciado un incremento en el riesgo de mortalidad en aquellos pacientes con antecedente de ERC y neumopatías (24-28) justificando el ingreso a la UCI cuando el índice de Charlson tiene un valor $\geq 3$ (29). Esto posiblemente debido a la alteración en el estado inmunológico presente en los pacientes con ERC, ya que éstos tienden a desarrollar compromiso leucocitario, defectos de la coagulación y estados de hipercoagulabilidad que conducen a mayor mortalidad en paciente con sepsis (30), similar a nuestro estudio donde hubo diferencias estadísticamente significativas entre la mortalidad en paciente sin ERC (8.9\%) vs aquellos que con ERC (19.8\%) con una oportunidad de $110 \%$ veces más de presentar mortalidad en AM con sepsis y ERC comparado con los AM son sepsis pero sin ERC.

La VGI es considerada la piedra angular de la práctica geriátrica, siendo una herramienta fundamental para el enfoque de los síndromes geriátricos los cuales pueden ser la primera manifestación de un proceso infeccioso en los AM (9) el modelo de trabajo en equipo interdisciplinario, la asistencia al paciente en diferentes niveles asistenciales (unidad de agudos, de media estancia, hospital de día, atención domiciliaria. Dentro de este grupo de síndromes se resalta el papel del delirium como un predictor de mortalidad. Pisani et al. reportaron que en AM de 60 años o más internados en la UCI, a mayor número de días con delirium, mayor riesgo de mortalidad al año siguiente de la admisión (HR 1.10; IC95\%, 1.02-1.18) (31); del mismo modo, en el metaanálisis 
publicado por Salluh et al. se demostró una mayor mortalidad (RR 2.2, IC95\% de 1.78-2.70; p<0.001), mayor duración de ventilación mecánica $(\mathrm{p}<0.001)$ y mayor estancia hospitalaria tanto en UCI ( $\mathrm{p}<0.001)$ como en hospitalización $(\mathrm{p}<0.001)$ en aquellos pacientes que desarrollaron delirium durante su estancia hospitalaria (32), teniendo además mayor mortalidad, hallazgo que fue observado en nuestro estudio donde los pacientes que desarrollaron delirium presentaron 2.1 veces más oportunidad de fallecer comparados con AM sin delirium.

Otra condición incluida en los análisis fue la presencia de la discapacidad evaluada mediante el IB. Tal como lo indicaron Wu LW et al. en su cohorte de 1834 pacientes entre los 60 y 84 años de edad en el 2016, demostró que la discapacidad aumenta dos veces el riesgo de muerte por cualquier causa (HR 2.3 (IC 95\%, 1.3-3.9, p<0.005) (33). De igual manera, otros estudios han demostrado la asociación entre la discapacidad y la mortalidad a largo plazo en pacientes hospitalizados con procesos infecciosos. Núria Torner et al. en un estudio retrospectivo de pacientes españoles hospitalizados por neumonía con edades entre 75 y 84 años, encontraron que los factores asociados significativamente con mortalidad a los 30 días fueron: tener un grado de discapacidad (OR 3.7, IC 95\%, 2.3-5.8; p <0.001), edad $\geq 85$ años (OR 3.0, IC 95\%, 1.7-5.3; p <0.001), función cognitiva alterada (OR 1.9, IC 95\%, 1.2-3.1; $\mathrm{p}=0.005)$ e ingreso en la UCI (OR 2.6, 95\% IC, 1.7-5.2; p = 0.009) (34). De igual manera, Cillóniz $\mathrm{C}$ et al. realizaron un estudio observacional prospectivo de 2149 pacientes adultos con neumonía durante un periodo de 12 años donde los resultados mostraron que la mortalidad aumenta con la edad (65-74 años, 6.9\%; 7584 años, $8.9 \%$; > 85 años, $17.1 \%$; $\mathrm{P}<.001)$ y se asoció con un aumento de las condiciones crónicas (neurológico que generaba discapacidad; OR, 2.1; IC 95\%; 1.5-2.1), índice de gravedad de la neumonía IV o V (OR, 3.2; IC del 95\%, 1.8-6.0), bacteriemia (OR, 1.7; IC del 95\%, 1.1-2.7) y la presencia de gérmenes multidrogorresistentes (OR, 2.4; $95 \%$ CI, 1.3-4.3) (35). Por otro lado, Quintero et al. en su estudio en pacientes AM hospitalizados por neumonía en una UGA registraron una letalidad a 30 días dl $26.3 \%$, en los cuales 93\% tenían una dependencia antes de la hospitalización en los fallecidos por neumonía comparado con AM sin dependencia, con diferencia estadísticamente significativa, indicando que los factores predictores independientes de la letalidad fueron: edad de 90 años o más (RR=1.6; IC95\%: 1.1-2,7; $\mathrm{p}=0.04)$, el compromiso multilobar $(\mathrm{RR}=1.9$; IC95\%: 1.1-3.3; $\mathrm{p}=0.02$ ), el nitrógeno ureico elevado (mediana $\geq 22.5 ; R R=3.9 ;$ IC95\%: 1.7-9,3; $<<0.01$ ), y un puntaje de cero en la escala de Lawton al ingreso $(R R=3.2$; IC95\% $1.1-9.8 ; \mathrm{p}=0.04)(36)$.

En relación con el proceso infeccioso los AM de 65 años son más proclives que los adultos jóvenes a cursar con infección por bacterias gramnegativa, ya que tienen 1.3 veces la oportunidad de verse afectados por estos microorganismos (12). Dentro de la familia de las Enterobacteriales, Esche- richia coli es el organismo más comúnmente identificado en los urocultivos obtenidos de pacientes con sepsis urinaria presentándose en un 50\% de los casos, pero existe la posibilidad que presenten otras bacterias gram-negativas como el Proteus spp, Klebsiella spp y Pseudomonas spp (37). Hallazgos semejantes a nuestro, estudio donde el agente causal más frecuente fue Escherichia coli en 46.6\%.

Por otro lado, en un estudio de cohorte prospectiva de AM encontró una tasa de mortalidad de $24 \%$ para pacientes con hemocultivos negativos y $31 \%$ en hemocultivos positivos (38) similar a nuestro estudio, siendo la principal causa de infección las de origen intraabdominal $(18,6 \%)$, seguido de neumonía asociada al cuidado de la salud y neumonía adquirida en la comunidad, con 17 y $12.4 \%$ respectivamente (39), siendo esta ultima la segunda causa de mortalidad en nuestro estudio.

A pesar de los reportes disponibles, la información sobre la epidemiología y el pronóstico de la sepsis en AM en nuestro medio es escasa, explicado en parte porque es una población excluida de la gran mayoría de las investigaciones $(3,12)$ a pesar de que ya se conoce a la edad como factor de riesgo predictor independiente para mortalidad, fallecimiento precoz tras el ingreso a hospitalización y ser institucionalizado $(5,12,40)$, lo que da más relevancia a nuestro estudio.

Dentro de las limitaciones esta su carácter retrospectivo que impide el análisis de otras variables que pudieran tener inferencia en el desenlace evaluado generando sesgo de información.

Como conclusión en nuestro estudio los AM hospitalizados por sepsis, los principales factores asociados con mortalidad a 30 días al ingreso de una UGA fueron la presencia de delirium, declinación funcional y ERC. Por lo tanto, es fundamental identificar e intervenir estos factores con el propósito de impactar en la mortalidad de los pacientes AM hospitalizado por sepsis.

\section{Fuentes de financiación}

Financiado por la Universidad Libre seccional Cali

\section{Referencias}

1. Rhodes A, Evans LE, Alhazzani W, Levy MM, Antonelli M, Ferrer R, et al. Surviving Sepsis Campaign: International Guidelines for Management of Sepsis and Septic Shock: 2016 [Internet]. Vol. 43, Intensive Care Medicine. 2017 [cited 2019 Oct 8].304-377 p. Available from: http://link.springer.com/10.1007/s00134017-4683-6

2. Rivers E, Nguyen B, Havstad S, Ressler J, Muzzin A, Knoblich B, et al. Early goal-directed therapy in the treatment of severe sepsis and septic shock. $N$ Engl J Med. 2001 Nov 8;345(19):1368-77.

3. Pinsky; DAL-ZLCC. Epidemiology of severe sepsis in the United States: Analysis of incidence, outcome, and associated costs of care. Crit Care Med. 2001;29(7):1303-10.

4. Kaukonen KM, Bailey M, Suzuki S, Pilcher D, Bellomo R. Mortality related to severe sepsis and septic shock among critically ill patients in Australia and New Zealand, 2000-2012. JAMA - J Am Med Assoc. 2014;311(13):1308-16.

5. Nasa P, Juneja D, Singh O, Dang R, Arora V. Severe sepsis and its impact on outcome in elderly and very elderly patients admitted in intensive care unit. Vol. 27, Journal of Intensive Care Medicine. 2012. p. 179-83.

6. Destarac LA, Ely W. Sepsis in Older Patients: An Emerging Concern in Critical Care. Adv SEPSIS. 2002;2. 
7. Nicholson K, Makovski TT, Griffith LE, Raina P, Stranges S, van den Akker M. Multimorbidity and comorbidity revisited: refining the concepts for international health research [Internet]. Vol. 105, Journal of Clinical Epidemiology. Elsevier USA; 2019 [cited 2020 Sep 9]. p. 142-6. Available from: http://www. jclinepi.com/article/S0895435618305432/fulltext

8. Martín S, Pérez A, Aldecoa C. Sepsis and immunosenescence in the elderly patient: A review. Vol. 4, Frontiers in Medicine. Frontiers Media S.A.; 2017.

9. González Montalvo JI, Alarcón Alarcón T. Grandes síndromes geriátricos. Concepto y prevención de los más importantes. Med - Programa Form Médica Contin Acreditado. 2003 Jan;8(108):5778-85.

10. Mayr FB, Yende S, Angus DC. Epidemiology of severe sepsis. Vol. 5, Virulence. Taylor and Francis Inc.; 2014. p. 1-11.

11. Deutschman CS, Tracey KJ. Sepsis: Current dogma and new perspectives. Vol. 40, Immunity. Cell Press; 2014. p. 463-75.

12. Martin GS, Mannino DM, Moss M. The effect of age on the development and outcome of adult sepsis. Crit Care Med. 2006;34(1):15-21.

13. Mouton CP, Bazaldua O V, Pierce B, Espino D V. Common infections in older adults. Am Fam Physician [Internet]. 2001 Jan 15 [cited 2019 Oct 8];63(2):257-68. Available from: http://www.ncbi.nlm.nih.gov/pubmed/11201692

14. Estadísticas Vitales. Boletín técnico (EEVV) [Internet]. 2018 [cited 2019 Oct 8]. Available from: https://www.dane.gov.co/files/investigaciones/poblacion/ bt_estadisticasvitales_2018pre-29-junio-2018.pdf

15. Seymour CW, Liu VX, Iwashyna TJ, Brunkhorst FM, Rea TD, Scherag A, et al. The third international consensus definitions for sepsis and septic shock (sepsis-3). JAMA - J Am Med Assoc. 2016; Feb 23;315(8):801-10.

16. Yarnall AJ, Sayer AA, Clegg A, Rockwood K, Parker S, Hindle J V. New horizons in multimorbidity in older adults. Age Ageing [Internet]. 2017 [cited 2020 Dec 16];46(6):882-8. Available from: /pmc/articles/PMC5860018/?report=abstract

17. Mahoney, F. I.Barthel DW. Functional evaluation: the barthel index. Md State Med J [Internet]. 1965 Feb 1 [cited 2020 Jun 22];14:61-5. Available from: https:// europepmc.org/article/med/14258950

18. Cid-Ruzafa J, Damián-Moreno J. Valoración de la discapacidad física: el índice de Barthel. Vol. 71, Re\ Esn Salud Púhhca. 1997.

19. OMS I Discapacidades. WHO [Internet]. 2016 [cited 2020 Dec 16]; Available from: http://www.who.int/topics/disabilities/es/

20. Inouye SK, Van Dyck CH, Alessi CA, Balkin S, Siegal AP, Horwitz RI. Clarifying confusion: The confusion assessment method: A new method for detection of delirium. Ann Intern Med [Internet]. 1990 Dec 1 [cited 2020 Jun 22];113(12):941-8. Available from: http://europepmc.org/article/med/2240918

21. Levey AS, Greene T, Sarnak MJ, Wang X, Beck GJ, Kusek JW, et al. Effect of Dietary Protein Restriction on the Progression of Kidney Disease: Long-Term Follow-Up of the Modification of Diet in Renal Disease (MDRD) Study. Am J Kidney Dis [Internet]. 2006 Dec 1 [cited 2020 Jun 22];48(6):879-88. Available from: http://www.ajkd.org/article/S0272638606013722/fulltext

22. KDIGO 2012. Clinical Practice Guideline for the Evaluation and Management of Chronic Kidney Disease. Off J Int Soc Nephrol [Internet]. 2013 [cited 2021 Aug 22];3(1). Available from: www.publicationethics.org

23. Holder AL, Gupta N, Lulaj E, Furgiuele M, Hidalgo I, Jones MP, et al. Predictors of early progression to severe sepsis or shock among emergency department patients with nonsevere sepsis. Int J Emerg Med [Internet]. 2016 Dec [cited 2019 Oct 10];9(1):10. Available from: http://www.ncbi.nlm.nih.gov/pubmed/26908009

24. Knaus WA, Wagner DP, Draper EA, Zimmerman JE, Bergner M, Bastos PG, et al. The APACHE III prognostic system: Risk prediction of hospital mortality for critically III hospitalized adults. Chest. 1991;100(6):1619-36.

25. Tran DD, Groeneveld AB, van der Meulen J, Nauta JJ, Strack van Schijndel RJ, Thijs LG. Age, chronic disease, sepsis, organ system failure, and mortality in a medical intensive care unit. Crit Care Med [Internet]. 1990 May [cited 2019 Oct 10];18(5):474-9. Available from: http://www.ncbi.nlm.nih.gov/pubmed/2328591
26. Pittet D, Thiévent B, Wenzel RP, Li N, Gurman G, Suter PM. Importance of pre-existing co-morbidities for prognosis of septicemia in critically ill patients. Intensive Care Med [Internet]. 1993 [cited 2019 Oct 10];19(5):265-72. Available from: http://www.ncbi.nlm.nih.gov/pubmed/8408935

27. Novosad SA, Sapiano MRP, Grigg C, Lake J, Robyn M, Dumyati G, et al. Vital Signs: Epidemiology of Sepsis: Prevalence of Health Care Factors and Opportunities for Prevention. MMWR Morb Mortal Wkly Rep [Internet]. 2016 Aug 26 [cited 2020 Sep 9];65(33):864-9. Available from: http://www.cdc.gov/mmwr/ volumes/65/wr/mm6533e1.htm

28. Doi K. Role of kidney injury in sepsis [Internet]. Vol. 4, Journal of Intensive Care BioMed Central Ltd.; 2016 [cited 2020 Sep 9]. Available from: /pmc/articles/ PMC4804517/?report=abstract

29. Oltean S, Tatulescu D, Bondor C, Slavcovici A, Cismaru C, Lupşe M, et al. Charlson's weighted index of comorbidities is useful in assessing the risk of death in septic patients. J Crit Care. 2012 Aug;27(4):370-5.

30.Bermejo-Martin J, Martín-Fernandez M, López-Mestanza C, Duque P, Almansa R. Shared Features of Endothelial Dysfunction between Sepsis and Its Preceding Risk Factors (Aging and Chronic Disease). J Clin Med [Internet]. 2018 Oct 30 [cited 2020 Sep 9];7(11):400. Available from: /pmc/articles/ PMC6262336/?report=abstract

31. Pisani MA, Kong SYJ, Kasl S V., Murphy TE, Araujo KLB, Van Ness PH Days of delirium are associated with 1-year mortality in an older intensive care unit population. Am J Respir Crit Care Med. 2009 Dec 1;180(11):1092-7.

32. Salluh JIF, Wang H, Schneider EB, Nagaraja N, Yenokyan G, Damluji A, et al. Outcome of delirium in critically ill patients: Systematic review and metaanalysis. BMJ. 2015 Jun 3;350:1-10.

33. Wu L-W, Chen W-L, Peng T-C, Chiang S-T, Yang H-F, Sun Y-S, et al. All-cause mortality risk in elderly individuals with disabilities: a retrospective observational study. [cited 2020 Apr 10]; Available from: http://bmjopen.bmj.com/

34. Torner N, Izquierdo C, Soldevila N, Toledo D, Chamorro J, Espejo E, et al. Factors associated with 30-day mortality in elderly inpatients with community acquired pneumonia during 2 influenza seasons. Hum Vaccines Immunother. 2017;13(2):450-5

35. Cillóniz C, Polverino E, Ewig S, Aliberti S, Gabarrús A, Menéndez R, et al. Impact of age and comorbidity on cause and outcome in community-acquired pneumonia. Chest [Internet]. 2013 Sep [cited 2019 Oct 10];144(3):999-1007. Available from: http://www.ncbi.nlm.nih.gov/pubmed/23670047

36. Quintero-González DC, Ocampo JM, Reyes-Ortiz CA. Factors associated with lethality from pneumonia in an acute care for the elderly unit: a retrospective cohort. Biomedica [Internet]. 2020 Dec 2 [cited 2020 Dec 21];40(4):734-48. Available from: http://www.ncbi.nlm.nih.gov/pubmed/33275351

37. Leligdowicz A, Dodek PM, Norena M, Wong H, Kumar A, Kumar A. Association between source of infection and hospital mortality in patients who have septic shock. Am J Respir Crit Care Med [Internet]. 2014 May 15 [cited 2020 Apr 10];189(10):1204-13. Available from: http://www.atsjournals.org/doi/abs/10.1164/ rccm.201310-1875OC

38. Jaimes, Fabian; Garces, Jenny; Cuervo, Jorge; Ramirez, Federico; Ramirez, Jorge; Quintero, Claudia; Vargas, Andrea; Zapata, Lader; Ochoa, Jorge; Yepes, Mercedes; Leal, Hiuber; Tandioy, Fabio; Estrada JC. NoFactores pronosticos en el sindrome de respuesta inflamatoria sistemica (SRIS): Desarrollo de un indice de severidad / Prognosis factors in SIRS. Acta méd colomb [Internet]. 2001 [cited 2019 Oct 8];26(4):149-57. Available from: http://www. actamedicacolombiana.com/anexo/articulos/04-2001-04.pdf

39. Ortíz G, Dueñas C, Rodríguez F, Barrera L, de La Rosa G, Dennis R, et al. Epidemiology of sepsis in Colombian intensive care units. Biomedica. 2014;34(1):40-7.

40. Azoulay E, Adrie C, De Lassence A, Pochard F, Moreau D, Thiery G, et al. Determinants of postintensive care unit mortality: a prospective multicenter study. Crit Care Med [Internet]. 2003 Feb [cited 2019 Oct 10];31(2):428-32. Available from: http://www.ncbi.nlm.nih.gov/pubmed/12576947 